\title{
Iris Nevus
}

National Cancer Institute

\section{Source}

National Cancer Institute. Iris Nevus. NCI Thesaurus. Code C4556.

A benign neoplasm that arises from the iris and is composed of melanocytes. Only a small percentage of cases progress to iris melanoma. 